\title{
Characteristics in Molecular Vibrational Frequency Patterns between Agonists and Antagonists of Histamine Receptors
}

\author{
S. June Oh* \\ Department of Pharmacology, Inje University College of Medicine, Busan 614-735, Korea
}

To learn the differences between the structure-activity relationship and molecular vibration-activity relationship in the ligand-receptor interaction of the histamine receptor, 47 ligands of the histamine receptor were analyzed by structural similarity and molecular vibrational frequency patterns. The radial tree that was produced by clustering analysis of molecular vibrational frequency patterns shows its potential for the functional classification of histamine receptor ligands.

Keywords: corralled intensity of molecular vibrational frequency, G protein-coupled receptors, histamine receptors, molecular vibration-activity relationship

\section{Introduction}

G protein-coupled receptors (GPCRs) are regarded to take up more than one-fourth of marketed human medicines [1, 2]. Drugs targeting GPCRs account for the majority of the best-selling drugs and about $40 \%$ of all prescription pharmaceuticals in the marketplace [3].

Histamine receptors belong to one family of rhodopsinlike class A GPCRs, and four subtypes are named in chronological order as $\mathrm{H}_{1}, \mathrm{H}_{2}, \mathrm{H}_{3}$, and $\mathrm{H}_{4}$. Histamine exerts its effects through the activation of these four histamine receptors. Each type of histamine receptor reacts to subtypespecific ligands into an active or inactive form.

GPCRs are integral membrane proteins that consist of 7 transmembrane segments connected by 3 intracellular and 3 extracellular loops of variable length. The crystal structures of GPCRs with their binding ligands have revealed the features of the ligand binding pockets and extracellular loops [4-6]. A ligand of GPCR activates the receptor by changing the receptor structure to the active form.

The biogenic amine histamine [2-(1H-imidazol-5-yl) ethanamine] (Fig. 1) is produced by decarboxylation of L-histidine and acts as a chemical mediator and neurotransmitter in central and peripheral tissues. It acts as an important pharmacological modulator involved in the processes of allergy, inflammation, neurophysiology, and cancer [7-10].

The molecular understanding of ligand-receptor interactions of GPCR remains unclear and is still the subject of investigations. There are several theories explaining the ligand-receptor interaction mechanism, such as shape theory, binding theory, and vibration theory $[11,12]$. The structures of histamine receptor ligands are so variable that we can not easily classify the pharmacological function of the ligand. To find any other characteristic in the molecular patterns between agonists and antagonists of histamine receptors, a computational approach to molecular vibration was carried out in an attempt to find a bit of the molecular interaction mechanisms.

\section{Methods}

\section{Dataset}

The simplified molecular-input line-entry system (SMILES) and 3-dimensional structure data format (SDF) files of the dataset were downloaded from the PubChem Compound Database in National Center for Biotechnology Information (NCBI) and used in the further analyses. All 47 
ligand molecules in the dataset, comprising 9 histamine agonists and 38 histamine antagonists, are shown in Table 1.

\section{Structure comparison of histamine receptor ligands}

The molecular similarities between histamine and other 46 chemicals were calculated from SMILES of the chemicals. The structural similarity was calculated and represented as the Tanimoto distance of each molecule from histamine. The Tanimoto coefficient for pairwise comparison of molecules is the most widely used measure of molecular structural similarity. This coefficient is defined as Tc $=N_{\mathrm{ab}} /\left(N_{\mathrm{a}}+N_{\mathrm{b}}-\right.$ $N_{\mathrm{ab}}$ ), with $N_{a b}$ being the number of common bits, $N_{a}$ the unique bits in molecule $a$, and $N_{b}$ the unique bits in molecule b, using a molecular fingerprint [13]. In this study, the molecular similarity was calculated as the Tanimoto coefficient using the 38-bit set.

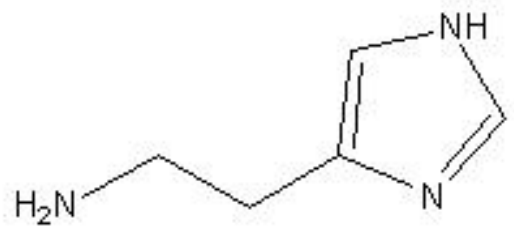

Fig. 1. The chemical structure of histidine.

\section{Geometry optimization and calculation of molecular vibrational frequency}

In order to calculate molecular vibrational frequency, the structure of a chemical must first be geometrically optimized. Since each provided theoretical 3-D conformer SDF is not at an energy minimum and may not represent the lowest energetic form in a vacuum, solvent, or a binding pocket, each SDF file of a ligand molecule underwent conversion to a single low-energy conformation using the general atomic and molecular electronic structure system (GAMESS) program package [14]. Restricted Hartree-Fock (RHF) calculations using Becke's exchange and Lee-Yang-Parr's correlation functionals (BLYP) density functional theory (DFT) method with 6-31G basis set were performed to optimize the geometries of the molecules. Each result was taken as the representative conformation of the molecule, although the calculation of molecular vibrational frequency has some dependence on conformation. Each geometry optimization result was subjected to the calculation step for the vibrational frequency with RUNTYP of HESSIAN in the GAMESS program.

Table 1. List of agonists and antagonists used in the present study

\begin{tabular}{|c|c|c|c|c|c|c|c|}
\hline PubChem ID & Compound name & Receptor & Function type & PubChem ID & Compound name & Receptor & Function type \\
\hline 87653 & 2-Thiazoleethanamine & $\mathrm{HRH} 1$ & Agonist & 3957 & Loratadine & $\mathrm{HRH} 1$ & Antagonist \\
\hline 2366 & Betahistine & $\mathrm{HRH} 1$ & Agonist & 4034 & Meclozine & $\mathrm{HRH} 1$ & Antagonist \\
\hline 75919 & Demethylbetahistine & $\mathrm{HRH} 1$ & Agonist & 4761 & Pheniramine & $\mathrm{HRH} 1$ & Antagonist \\
\hline 7741 & Betazole & $\mathrm{HRH} 2$ & Agonist & 4927 & Promethazine & $\mathrm{HRH} 1$ & Antagonist \\
\hline 3077 & Dimaprit & $\mathrm{HRH} 2$ & Agonist & 5002 & Quetiapine & $\mathrm{HRH} 1$ & Antagonist \\
\hline 3692 & Imetit & $\mathrm{HRH} 3$ & Agonist & 3032915 & Burimamide & $\mathrm{HRH} 2$ & Antagonist \\
\hline 126688 & Amthamine & $\mathrm{HRH} 3, \mathrm{H} 4$ & Agonist & 2756 & Cimetidine & $\mathrm{HRH} 2$ & Antagonist \\
\hline 41376 & Impromidine & $\mathrm{HRH} 4$ & Agonist & 5282136 & Lafutidine & $\mathrm{HRH} 2$ & Antagonist \\
\hline 5227 & SKF91488 & $\mathrm{HR}$ & Agonist & 3033637 & Nizatidine & $\mathrm{HRH} 2$ & Antagonist \\
\hline 2267 & Azelastine & $\mathrm{HRH} 1$ & Antagonist & 5282450 & Pibutidine & $\mathrm{HRH} 2$ & Antagonist \\
\hline 2678 & Cetirizine & $\mathrm{HRH} 1$ & Antagonist & 3001055 & Ranitidine & $\mathrm{HRH} 2$ & Antagonist \\
\hline 2725 & Chlorpheniramine & $\mathrm{HRH} 1$ & Antagonist & 5105 & Roxatidine & $\mathrm{HRH} 2$ & Antagonist \\
\hline 26987 & Clemastine & $\mathrm{HRH} 1$ & Antagonist & 50287 & Tiotidine & $\mathrm{HRH} 2$ & Antagonist \\
\hline 6726 & Cyclizine & $\mathrm{HRH} 1$ & Antagonist & 9954017 & A-349821 & $\mathrm{HRH} 3$ & Antagonist \\
\hline 124087 & Desloratadine & $\mathrm{HRH} 1$ & Antagonist & 9818903 & ABT-239 & $\mathrm{HRH} 3$ & Antagonist \\
\hline 33036 & Dexchlorpheniramine & $\mathrm{HRH} 1$ & Antagonist & 2366 & Betahistine & $\mathrm{HRH} 3$ & Antagonist \\
\hline 21855 & Dimetindene & $\mathrm{HRH} 1$ & Antagonist & 6422124 & Ciproxifan & $\mathrm{HRH} 3$ & Antagonist \\
\hline 3100 & Diphenhydramine & $\mathrm{HRH} 1$ & Antagonist & 3035746 & lodophenpropit & $\mathrm{HRH} 3$ & Antagonist \\
\hline 667477 & Doxepin & $\mathrm{HRH} 1$ & Antagonist & 9948102 & Pitolisant & $\mathrm{HRH} 3$ & Antagonist \\
\hline 3162 & Doxylamine & $\mathrm{HRH} 1$ & Antagonist & 3035905 & Thioperamide & $\mathrm{HRH} 3, \mathrm{H} 4$ & Antagonist \\
\hline 3191 & Ebastine & $\mathrm{HRH} 1$ & Antagonist & 2790 & Clobenpropit & $\mathrm{HRH} 3$ & Antagonist \\
\hline 19105 & Embramine & $\mathrm{HRH} 1$ & Antagonist & 4908365 & JNJ-7777120 & $\mathrm{HRH} 4$ & Antagonist \\
\hline 3348 & Fexofenadine & $\mathrm{HRH} 1$ & Antagonist & 10446295 & VUF6002 & $\mathrm{HRH} 4$ & Antagonist \\
\hline 1549000 & Levocetirizine & $\mathrm{HRH} 1$ & Antagonist & & & & \\
\hline
\end{tabular}




\section{Hierarchical clustering of the corralled intensity of molecular vibrational frequency (CIMVF)}

For the simplified molecular comparison, the calculated vibrational frequencies of a molecule were then sorted in increasing order and taken into the corrals, the step size of which was 5 . The intensities of each frequency in the same corral were summed up as the representative of the corral in the frequency range of $0-5,000 \mathrm{~cm}^{-1}$. As a final outcome, this potential molecular descriptor of each molecule was displayed in a 1-dimensional vector containing 1,000 elements. Finally, the similarity matrix, comprising the descriptors of 47 ligands of histamine receptor, was then subjected to hierarchical clustering in the agglomerative manner. In this study, the similarity matrix was finally clustered to make an unrooted tree of 47 vertices. The calculations of CIMVF were performed by in-house scripts, written in Python.

\section{Results and Discussion}

The Tanimoto coefficients between histamine and other ligand molecules are shown in Table 2. Typically, a Tanimoto coefficient $>0.85$ is considered highly similar, and a coefficient $>0.75$ is considered similar for the purpose of clustering molecules that may have similar biological activity profiles [15]. The highest Tanimoto coefficient among agonists was 0.45 (imetit), and this is not high enough to be considered a molecule that has potential agonist efficacy. Moreover, the lowest value of Tanimoto coefficient among agonists was 0.08 (dimaprit and SKF91488) and is also a lower value as an antagonist. It seems that there is no related pattern between Tanimoto coefficients and the functional types of molecules in the case of histamine receptor agonists or antagonists.

To search for a novel characteristic for the classification of histamine receptor ligands, a kind of molecular calculation using agglomerative hierarchical clustering was adopted in this work. The result of the hierarchical clustering of the similarity matrix from CIMVF is shown in Fig. 2. As shown in the figure, eight agonists were located nearby (the part in the dotted circle), except impromidine, and all antagonists were clustered close to each other in the radial tree. We can tell the regional difference between agonists and antagonists in the tree and also find that the information from the molecular vibrational frequency may play a role in the classification of agonists/antagonists for histamine receptor as a possible molecular descriptor. For these methods, clustering with CIMVF shows the more proper result in the

Table 2. The molecular similarity between histamine and each ligand molecule in Tanimoto coefficient

\begin{tabular}{|c|c|c|c|c|c|}
\hline Compound & Tanimoto coefficient & Function type & Compound & Tanimoto coefficient & Function type \\
\hline Histamine & 1 & Agonist & & & \\
\hline Imetit & 0.45 & Agonist & Loratadine & 0.13 & Antagonist \\
\hline Burimamide & 0.43 & Antagonist & JNJ-7777120 & 0.13 & Antagonist \\
\hline Thioperamide & 0.41 & Antagonist & Pitolisant & 0.12 & Antagonist \\
\hline Iodophenpropit & 0.3 & Antagonist & Embramine & 0.12 & Antagonist \\
\hline Demethylbetahistine & 0.3 & Agonist & Cyclizine & 0.11 & Antagonist \\
\hline Ciproxifan & 0.3 & Antagonist & Dimetindene & 0.11 & Antagonist \\
\hline Impromidine & 0.29 & Agonist & Meclozine & 0.11 & Antagonist \\
\hline Clobenpropit & 0.29 & Antagonist & Nizatidine & 0.1 & Antagonist \\
\hline Betahistine & 0.27 & Antagonist & Promethazine & 0.1 & Antagonist \\
\hline Amthamine & 0.25 & Agonist & Azelastine & 0.1 & Antagonist \\
\hline 2-Thiazoleethanamine & 0.25 & Agonist & Cetirizine & 0.1 & Antagonist \\
\hline Cimetidine & 0.24 & Antagonist & Levocetirizine & 0.1 & Antagonist \\
\hline Betazole & 0.22 & Agonist & Tiotidine & 0.09 & Antagonist \\
\hline VUF6002 & 0.19 & Antagonist & ABT-239 & 0.09 & Antagonist \\
\hline Doxylamine & 0.18 & Antagonist & Doxepin & 0.09 & Antagonist \\
\hline Pheniramine & 0.18 & Antagonist & Dimaprit & 0.08 & Agonist \\
\hline Chlorpheniramine & 0.18 & Antagonist & A-349821 & 0.08 & Antagonist \\
\hline Dexchlorpheniramine & 0.18 & Antagonist & Pibutidine & 0.08 & Antagonist \\
\hline Fexofenadine & 0.16 & Antagonist & SKF91488 & 0.08 & Agonist \\
\hline Desloratadine & 0.14 & Antagonist & Roxatidine & 0.08 & Antagonist \\
\hline Diphenhydramine & 0.14 & Antagonist & Ranitidine & 0.07 & Antagonist \\
\hline Clemastine & 0.13 & Antagonist & Lafutidine & 0.07 & Antagonist \\
\hline Ebastine & 0.13 & Antagonist & Quetiapine & 0.07 & Antagonist \\
\hline
\end{tabular}

The Tanimoto coefficients between histamine and other ligand molecules are listed in descending order. 


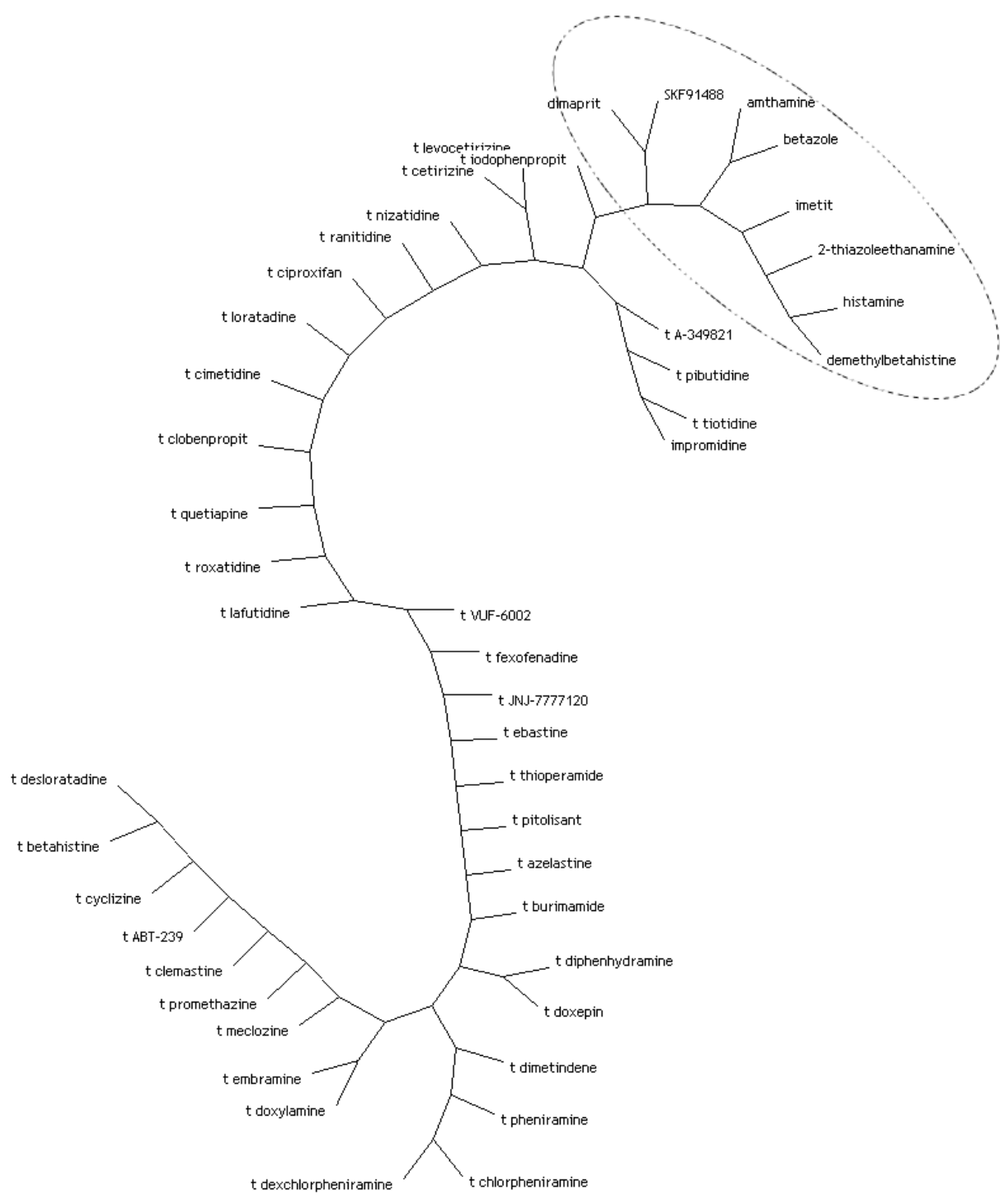

Fig. 2. Radial tree of corralled intensity of molecular vibrational frequency (CIMVF) clustering using the complete linkage method. Antagonists are tagged with " $\mathrm{t}$ " to their chemical names as a prefix, whereas agonists are not. Agonists of histamine receptors except impromidine are located in a cluster (the part in the dotted circle). case of histamine receptor ligands. With a more concentrated study on the relationship between the molecular vibrational frequency and pharmacological function of a ligand, the vibrational spectrum of a molecule may shed light on the field of ligand-receptor interaction mechanisms.

\section{Acknowledgments}

The author would like to express sincere appreciation to Prof. C. H. Choi for the use of his cluster computer and his valuable advice. This work was supported by the 2010 Inje University research grant.

\section{References}

1. Bleicher KH, Bohm HJ, Müller K, Alanine AI. Hit and lead gen- eration: beyond high-throughput screening. Nat Rev Drug Discov 2003;2:369-378.

2. Zheng CJ, Han LY, Yap CW, Ji ZL, Cao ZW, Chen YZ. Therapeutic targets: progress of their exploration and investigation of their characteristics. Pharmacol Rev 2006;58:259279.

3. Drews J. Drug discovery: a historical perspective. Science 2000;287:1960-1964.

4. Cherezov V, Rosenbaum DM, Hanson MA, Rasmussen SG, Thian FS, Kobilka TS, et al. High-resolution crystal structure of an engineered human beta2-adrenergic $\mathrm{G}$ protein-coupled receptor. Science 2007;318:1258-1265.

5. Chien EY, Liu W, Zhao Q, Katritch V, Han GW, Hanson MA, et al. Structure of the human dopamine D3 receptor in complex with a D2/D3 selective antagonist. Science 2010;330:10911095.

6. Shimamura T, Shiroishi M, Weyand S, Tsujimoto H, Winter G, Katritch V, et al. Structure of the human histamine H1 receptor 
complex with doxepin. Nature 2011;475:65-70.

7. Medina VA, Rivera ES. Histamine receptors and cancer pharmacology. Br J Pharmacol 2010;161:755-767.

8. Schwartz JC, Arrang JM, Garbarg M, Pollard H, Ruat M. Histaminergic transmission in the mammalian brain. Physiol Rev 1991;71:1-51.

9. Hill SJ. Distribution, properties, and functional characteristics of three classes of histamine receptor. Pharmacol Rev 1990;42:45-83.

10. Hill SJ, Ganellin CR, Timmerman H, Schwartz JC, Shankley NP, Young JM, et al. International Union of Pharmacology. XIII. Classification of histamine receptors. Pharmacol Rev 1997;49:253-278.

11. Keller A, Vosshall LB. A psychophysical test of the vibration theory of olfaction. Nat Neurosci 2004;7:337-338.

12. Takane SY, Mitchell JB. A structure-odour relationship study using EVA descriptors and hierarchical clustering. Org Biomol Chem 2004;2:3250-3255.

13. Godden JW, Xue L, Bajorath J. Combinatorial preferences affect molecular similarity/diversity calculations using binary fingerprints and Tanimoto coefficients. J Chem Inf Comput Sci 2000;40:163-166.

14. Schmidt MW, Baldridge KK, Boatz JA, Elbert ST, Gordon MS, Jensen $\mathrm{JH}$, et al. General atomic and molecular electronic structure system. J Comput Chem 1993;14:1347-1363.

15. Keseru GM, Makara GM. The influence of lead discovery strategies on the properties of drug candidates. Nat Rev Drug Discov 2009;8:203-212. 